\title{
Unexpected point mutations activate cryptic 3' splice sites by perturbing a natural secondary structure within a yeast intron
}

\author{
James O. Deshler ${ }^{1,2}$ and John J. Rossi ${ }^{2,3}$ \\ ${ }^{1}$ Department of Microbiology, University of California at Los Angeles, Los Angeles, California 90024 USA; ${ }^{2}$ Department of \\ Molecular Genetics, Beckman Research Institute, City of Hope, Duarte, California 91010 USA
}

The $3^{\prime}$ splice site of the budding yeast Kluyveromyces lactis actin gene (ACT) intron is distally spaced (122 nucleotides) from its branchpoint and is also preceded by a silent PyAG located 43 nucleotides upstream. We devised a genetic screen that resulted in the isolation of several randomly induced cis-acting mutations that activate the silent PyAG as a $3^{\prime}$ splice site. These mutations fall within a region surrounding this PyAG, which can hypothetically fold into a higher-order structure. Site-directed mutational analyses demonstrate that a hairpin structure in this region is required for correct $3^{\prime}$ splice-site selection. Analysis of the point mutations suggests that local breathing of the hairpin near the first PyAG can lead to its activation. These data demonstrate that $3^{\prime}$ splice-site selection is not a consequence of a linear, directional scanning mechanism, but support the notion of a critical positioning requirement for 3 ' splice-site selection. We speculate on the possible origin of this intron-encoded structural motif, which has homology to a bacterial transposon and suggests one possible origin for alternative splicing mechanisms in higher eukaryotes.

[Key Words: Kluyveromyces lactis; intron structure; 3' splice site; branchpoint; point mutations]

Received January 7, 1991; revised version accepted April 2, 1991.

The splicing of all known nuclear pre-mRNAs requires a spliceosome and is characterized by a two-step chemical reaction. In the first step, exon I is cleaved with the concomitant formation of a lariat molecule. In the second step, the intron is excised by cleavage at the $3^{\prime}$ splice site and exon $I$ is simultaneously ligated to exon II (Domdey et al. 1984; Padgett et al. 1984; Ruskin et al. 1984; Zeitlin and Efstratiadis 1984). Sequence inspection of all known Saccharomyces cerevisiae introns reveals highly conserved sequences at the $5^{\prime}$ splice site (GUAUGU), branchpoint (UACUAAC), and 3' splice site (PyAG). Mutational analyses and molecular characterizations have verified that these sequences are required for splicing and are recognized by spliceosomal components during spliceosome assembly on the pre-mRNA substrate (Rymond and Rosbash 1985; Vijayraghavan et al. 1986; Parker et al. 1987; Seraphin et al. 1988; Siliciano and Guthrie 1988).

Several laboratories have investigated the role of nonconserved intron sequences in the splicing process. In yeast it has been demonstrated that splicing efficiencies can be affected by mutations outside of the conserved sequences (Pikielny and Rosbash 1985; Newman 1987).

${ }^{3}$ Corresponding author.
In a mammalian system it has been shown that the sequence environment of a splice site can affect the relative efficiency of its usage when compared with a ciscompeting site (Reed and Maniatis 1986). The mechanism by which nonconserved intron sequences affect splicing efficiencies or alternative splice-site selection is poorly understood. One possible mechanism is RNA secondary structure, which has been shown to affect splicesite selection in several systems (Solnick 1985; Eperon et al. 1988; Chebli et al. 1989; Yoshimatsu and Nagawa 1989) and has been hypothesized as a possible mechanism for alternative splicing of the rat preprotachykinin and $\beta$-tropomyosin genes (Helfman et al. 1990; Libri et al. 1990; Nasim et al. 1990).

The choice of $3^{\prime}$ splice sites in yeast is believed to be dictated by the position of branch formation. Langford and Gallwitz (1983) showed that when random sequences were placed downstream of the yeast branchpoint sequence, the $3^{\prime}$ splice site was defined as the first AG 3' of the branchpoint. Subsequently, Fouser and Freisen (1987) found that no critical bases between the branchpoint and 3' splice site were required for splicing the $S$. cerevisiae actin gene $(\mathrm{ACT})$ pre-mRNA. Finally, it was shown that extending the distance between the branchpoint and AG to 110 nucleotides in the S. cerevi- 
siae ACT intron virtually eliminated the second step of the splicing reaction (Cellini et al. 1986). Therefore, it is thought that in yeast, the first AG (usually a PyAG) downstream of the branchpoint is chosen as long as it is not positioned too far from the branchpoint. This is consistent with a $5^{\prime} \rightarrow 3^{\prime}$ molecular-scanning mechanism recently proposed for mammalian $3^{\prime}$ splice-site selection (Smith et al. 1989).

Recently, we showed that the Kluyveromyces lactis ACT intron contains an unusual 3' splice site (Deshler et al. 1989). In this intron, a PyAG (AG1), located 73 nucleotides downstream of the branchpoint, is skipped, and the second AG (AG2), positioned 122 nucleotides downstream of the branchpoint, is chosen as a $3^{\prime}$ splice site. The organization of this intron poses two questions that cannot be answered by previously proposed models for $3^{\prime}$ splice-site selection in yeast: (1) Why is the first AG skipped? and (2) How does the distal AG overcome a distance from the branchpoint, which was previously shown to be inhibitory to the splicing reaction? We describe the first genetic screen, to our knowledge, that allows the identification of point mutations that activate usage of a suppressed splice site (AG1). By fusing the Escherichia coli lacZ gene downstream and in the reading frame of the first $A G$, we were able to monitor the efficiency of splicing to AG1 by observing colony color on X-gal-containing plates. Random mutagenesis of the region between the branchpoint and the $3^{\prime}$ splice site, and subsequent screening for increased $\beta$-galactosidase activity demonstrate that nonconserved sequence information within the intron defines the correct 3 ' splice site. Further analyses using site-directed mutagenesis provide proof that specific secondary structural interactions position the distal AG for proper $3^{\prime}$ splice-site selection, thus overcoming structural constraints that would ordinarily not allow usage of a splice site. Our data demonstrate that nonconserved sequences within pre-mRNA introns can influence alternative splice-site selection via structural motifs, supporting the idea that pre-mRNA introns can be highly evolved molecules with functional and structural constraints. These results have significant implications for the regulation of alternative splicing in higher eukaryotes.

\section{Results}

Description of the K. lactis 3 ' splice site

Figure 1 shows the sequence of the wild-type $K$. lactis ACT intron downstream of and including the branchpoint (UACUAAC). Important features of this sequence are the presence of a naturally occurring EcoRI site located 29 nucleotides $3^{\prime}$ of the branchpoint, a PyAG (AG1) located 73 nucleotides downstream of the branchpoint, and the $3^{\prime}$ splice site (AG2) located 122 nucleotides downstream of the branchpoint. In addition, two reading frames are depicted (RF1 and RF2), which correspond to splicing of exon I to AG1 or AG2, respectively. Note that two stop codons in RF1 are present 6 and 27 nucleotides $3^{\prime}$ of AG1. These codons would terminate translation of mRNA molecules produced by utilization of AG1 as a $3^{\prime}$ splice site.

\section{AG1 is used as a $3^{\prime}$ splice site at low efficiency by} both $\mathrm{S}$. cerevisiae and $\mathrm{K}$. lactis

The $5^{\prime}$ region of the $K$. lactis ACT gene, including the intron and only two nucleotides of exon II, was fused to the E. coli lacZ gene in the reading frame of AGl (RF1) and transformed into $S$. cerevisiae. The stop codons (positions 79 and 100 in Fig. 1) were converted to amino acid codons, and S. cerevisiae transformants harboring this construct gave rise to colonies that scored weakly positive for $\beta$-galactosidase activity after 3-5 days of incubation on X-gal indicator plates at $30^{\circ} \mathrm{C}$ (data not shown), suggesting that AG1 can be used at low efficiency as a 3' splice site.

Because $S$. cerevisiae appeared to use AG1 of the $K$. lactis ACT intron as an extremely weak $3^{\prime}$ splice site, we wanted to determine whether $K$. lactis utilized AGl similarly as a $3^{\prime}$ splice site in the endogenous ACT transcript. To examine this, a primer complementary to the last 22 nucleotides of the $K$. lactis ACT intron and a second primer located upstream of the ACT exon I (Fig. 2B) were designed to amplify specifically aberrantly spliced RNAs in an RNA-dependent polymerase chain reaction (PCR) assay (Saiki et al. 1988; Deshler et al. 1989|. These primers cannot amplify the most abundant,

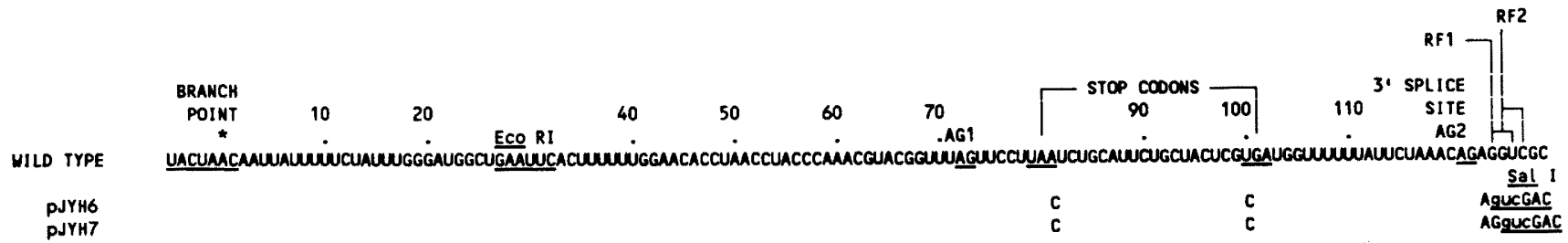

Figure 1. RNA sequence of the $K$. lactis ACT intron in the region of the $3^{\prime}$ splice site. Important features are the branchpoint signal (UACUAAC), EcoRI site, AG1, AG2, and two stop codons in the reading frame of AG1. Below the sequence are the nucleotide changes used to create plasmids pJYH6 and pJYH7. The first 3 nucleotides of the Sall site (signified by lowercase letters) are in the lacZ reading frame of the yeast shuttle vector p2UB. As can be seen, they are positioned in RF1 or RF2 for pJYH6 and pIYH7, respectively. All subsequent figures use the indicated numbering scheme for identifying nucleotide positions. 


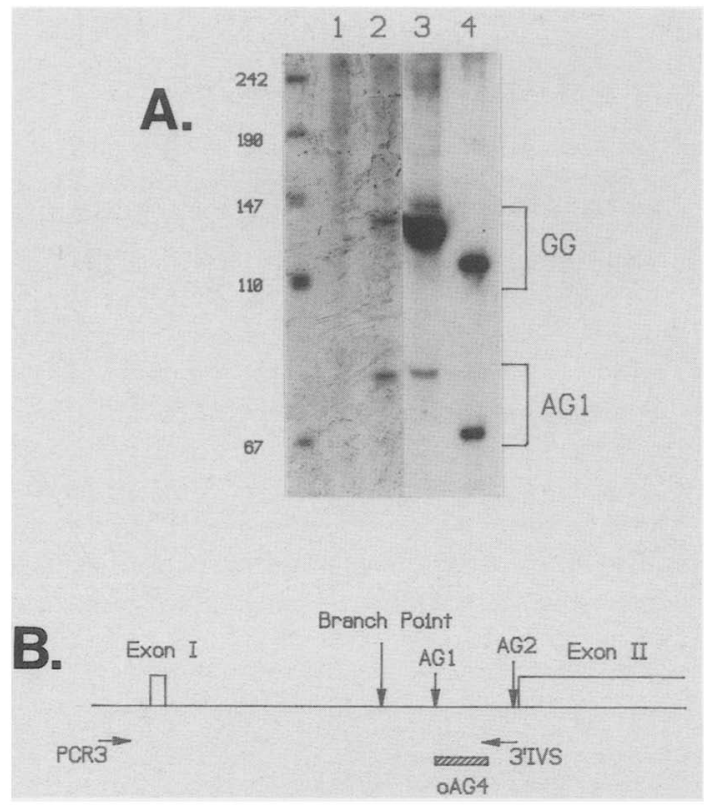

Figure 2. S. cerevisiae and $K$. lactis use AGl as a $3^{\prime}$ splice site at low efficiency. (A) RNA-dependent PCR assays were carried out using the primers and probe described below (in $B$ ) on $100 \mathrm{ng}$ of total RNA prepared from $K$. lactis with the reverse transcriptase step omitted as a control (lane 1), K. lactis (lane 2), $S$. cerevisiae transformed with pCVBB that contains the $5^{\prime}$ half of the $K$. lactis ACT gene (lane 3 ), and S. cerevisiae transformed with pJYH6 using the SCE-IS $5^{\prime}$ primer indicated in Fig. 6 (lane 4) (for details of GG and AG1 in S. cerevisiae and K. lactis, see text). (B) Diagramatic representation of the $K$. lactis ACT target gene, amplification primers (PCR3 and 3'IVS), and detection probe (oAG4). PCR3 (5'-CTTCACGCGCTATAGTATAAC-3') is specific for the $K$. lactis ACT $5^{\prime}$-untranslated region, $3^{\prime}$ IVS (5' -CTGTTTAGAATAAAAAACCATC- $\left.3^{\prime}\right)$ is complementary to the last 22 nucleotides of the $K$. lactis ACT intron, and the sequence of oAG4 is given in Materials and methods.

properly spliced ACT mRNA and therefore allow for specific amplification and detection of minor, aberrantly spliced products. In lane 2 of Figure 2A, an 80-nucleotide product from $K$. lactis total RNA, which is precisely the size expected if AGl were utilized as a $3^{\prime}$ splice site, is detected. This product was purified and sequenced to verify its identity (data not presented). Figure 2A (lane 3) shows that AGl is also used when the K. lactis ACT intron and 240 nucleotides of exon II (plasmid pCVBB) are expressed in $S$. cerevisiae, confirming the results obtained with the K. lactis ACT-E. coli lacZ gene fusion.

\section{A GG dinucleotide located 19 nucleotides downstream of the branchpoint is also a competing $3^{\prime}$ splice site}

In addition to the 80-nucleotide product, a 134-nucleotide product is seen in both lanes 2 and 3 of Figure 2A. This corresponds to a spliced product in which the GG at position 19 (Fig. 1) serves as a $3^{\prime}$ splice site. Because this was an unexpected product, we purified and sequenced the PCR-amplified DNA. The sequence obtained (Fig. 3) demonstrates that the GG located at position 19 is used as a $3^{\prime}$ acceptor site. The fact that this GG is preceded by a polyuridine tract and that it is positioned close to the branchpoint might explain why it is used aberrantly as a $3^{\prime}$ splice site in S. cerevisiae (see Discussion). Although $K$. lactis utilizes this aberrant splice site with the same efficiency as it uses AGl, $S$. cerevisiae transformed with pCVBB uses it at a higher relative frequency /cf. the intensity of the GG and AG1 products in lanes 2 and 3 of Fig. 2A). Because the GG is followed by an in-frame stop codon (position 50, Fig. 1), its use does not generate the LAC phenotype used in the genetic screen described below. This screen is designed to isolate mutations between the branchpoint and AG2, which disrupt the normal splicing pattern of this intron.

\section{All cis-encoded information required for proper splice site selection is located between the branchpoint and 3' splice site}

To determine whether all of the information required for $3^{\prime}$ splice-site selection is located between the branch-

Figure 3. The GG at position 19 is used aberrantly as a $3^{\prime}$ splice site. The sequence of the PCR product seen in Fig. 2A, lane 4, which corresponds to a splice using the GG dinucleotide at position 19, is illustrated. The product was gel-purified and sequenced as described in Materials and methods. Exon $I$ is spliced 7 nucleotides upstream of the EcoRI site, consistent with a splice to the indicated GG.

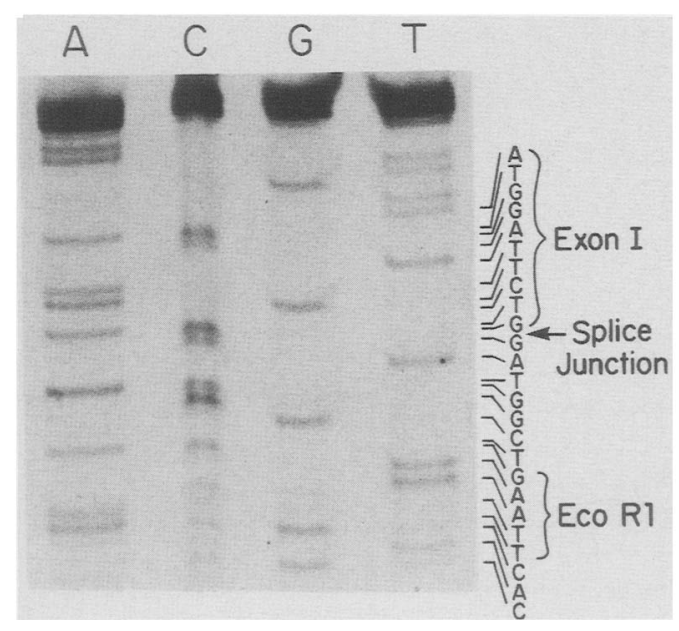


point and the $3^{\prime}$ splice site, a tripartite hybrid gene was constructed (Fig. 4), in which the entire sequence $5^{\prime}$ of the branchpoint sequence, including the promoter and exon I, is from the $S$. cerevisiae ACT gene. All nucleotides downstream of the branchpoint are from the $K$. lactis ACT gene. This includes the $3^{\prime}$ splice site, which is fused to the E. coli lacZ gene in one of two reading frames (RF1 or RF2; Fig. 1). $\beta$-Galactosidase expression is therefore dependent on a splice to AG1 or AG2, respectively, and splicing is monitored by assaying $\beta$-galactosidase activity of $S$. cerevisiae transformants harboring either plasmid pJYH6 (for AG1) or pJYH7 (for AG2) (Fig. 1). Both of these plasmids also have the two intron-encoded stop codons modified to amino acid codons (Fig. 1). These alterations do not affect the normal splicing pattern (Fig. 2A, lane 4 for pJYH6; data not presented for pJYH7). One to three units of $\beta$-galactosidase activity are produced from pJYH6 in which lacZ is translationally in-frame with AG1. In contrast, pIYH7 directs the production of $150 \beta$-galactosidase units, suggesting that the ratio of AG1 to AG2 usage is $\sim 1: 100$ in S. cerevisiae. These results show that AG2 is highly preferred to AG1 as a $3^{\prime}$ splice signal, even when the $3^{\prime}$ region of the $K$. lactis intron is placed downstream of the $S$. cerevisiae ACT branchpoint sequence. The data presented in Figure 2A, lane 4, and Figure 7, lane C (below), demonstrate that the aberrant splice signal GG at position 19 is again used preferentially over AG1 in $S$. cerevisiae expressing the hybrid intron.

\section{Random mutagenesis identifies nonconserved nucleotides important for $3^{\prime}$ splice-site selection}

Because pJYH6 produces one to three units of $\beta$-galactosidase activity, $S$. cerevisiae transformants give rise to faint blue colonies on X-gal indicator plates after 2-3 days of incubation at $30^{\circ} \mathrm{C}$. Plasmid $\mathrm{pJYH} 7$ produces 150 units of $\beta$-galactosidase activity, and colonies turn dark blue within $12 \mathrm{hr}$. Taking advantage of this difference, we devised a genetic screen to identify nonconserved nucleotides required for correct 3 ' splice-site selection. The sequence located between the branchpoint and AG2 was randomly mutagenized in pJYH6, which has the lacZ gene in the reading frame of AGl. The resulting mutant

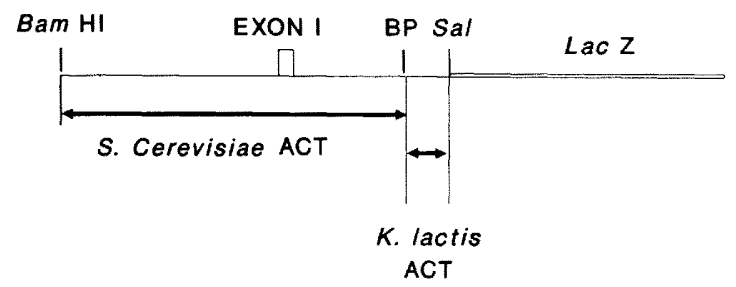

Figure 4. S. cerevisiae ACT/K. lactis ACT/E. coli lacZ tripartite gene of pIYH6 and pJYH7. A combination of site-directed and PCR mutageneses was used to create a chimeric gene fusing the indicated regions of $S$. cerevisiae ACT, K. lactis ACT, and lacZ. The construction is described in Materials and methods. (BP) Branchpoint sequence (UACUAAC). plasmid pool was used to transform $S$. cerevisiae strain JM43. Individual transformants $(\sim 3000)$ were patched onto X-gal plates. Colonies that appeared blue before the pJYH6 harboring control were isolated and assayed for $\beta$-galactosidase activity. Nine independently isolated clones (representing seven different mutations) were identified as having increased $\beta$-galactosidase activity relative to the parental control (pJYH6). Plasmid DNAs from these colonies were isolated, and the region of interest was sequenced.

Each clone had at least one nucleotide change between the branchpoint and the $3^{\prime}$ splice site (Fig. 5). Two classes of mutations were identified. Class I mutants (AG03 and AG10) resulted from the generation of a new $A G$ close to $A G 2$, but in the reading frame of AG1. For example, mutant AG03 has an A $\rightarrow G$ transition located 5 nucleotides upstream of AG2 (Fig. 5). Molecular analysis shows that the new AG is the major $3^{\prime}$ splice site giving rise to a PCR product that migrates slightly slower than the pJYH6 AG2 product (Fig. 6). Mutant AG10 (isolated independently twice) has a $G \rightarrow U$ transversion in AG2 (Fig. 5), suggesting that the AG located 2 nucleotides downstream of AG2 is used as a $3^{\prime}$ splice site. This AG is in the reading frame of AGl, which explains the positive scoring of these mutants in the $\mathrm{X}$ gal screen.

Class II was the class of mutations that was sought after because the increase in $\beta$-galactosidase activity could not be explained by the creation of a new AG, a shift in reading frame, or the translation of an mRNA that resulted from a splice to the upstream GG (position 19, Fig. 1). The most likely explanation for the increase in $\beta$-galactosidase activity in these mutants is an increased utilization of AGl as a $3^{\prime}$ splice site. Figure 5 shows the sequence changes and the relative increases in $\beta$-galactosidase activities. Mutant AG04, which was isolated independently twice, shows the largest increase in $\beta$-galactosidase activity. This mutation is a deletion of a single $\mathrm{G}$ at position 41 or 42 . AG21 is a double mutant $A(87)$ to $G$ and $C(90)$ to $U$, which increases $\beta$-galactosidase activity approximately fourfold, as does AG36, which contains an $\mathrm{A} \rightarrow \mathrm{G}$ transition at position 51 . AG53 and AG65 are also double mutants that increase enzyme activity 7 - and 10 -fold, respectively.

To assess the overall pattern of $3^{\prime}$ splice-site selection in the various mutants, total RNAs were prepared and analyzed by RNA-dependent PCRs. In our hands, this assay qualitatively reflects the relative preference of the three competing $3^{\prime}$ splice sites. Figure 6 illustrates that mutants AG04, AG36, AG53, and AG65 all have an increased level of the AGl splice-site product (127 nucleotides) relative to the parental construct pJYH6. Also, the level of GG usage is affected to varying degrees in some of the mutants. The most extreme example of this effect is mutant AG04, which in addition to having the highest AG1 activation (14-fold as determined by $\beta$-galactosidase assay), clearly favors the GG over AG2 as a $3^{\prime}$ splice site, demonstrating that a single point mutation can dramatically influence the relative usage of all three acceptor sites. 


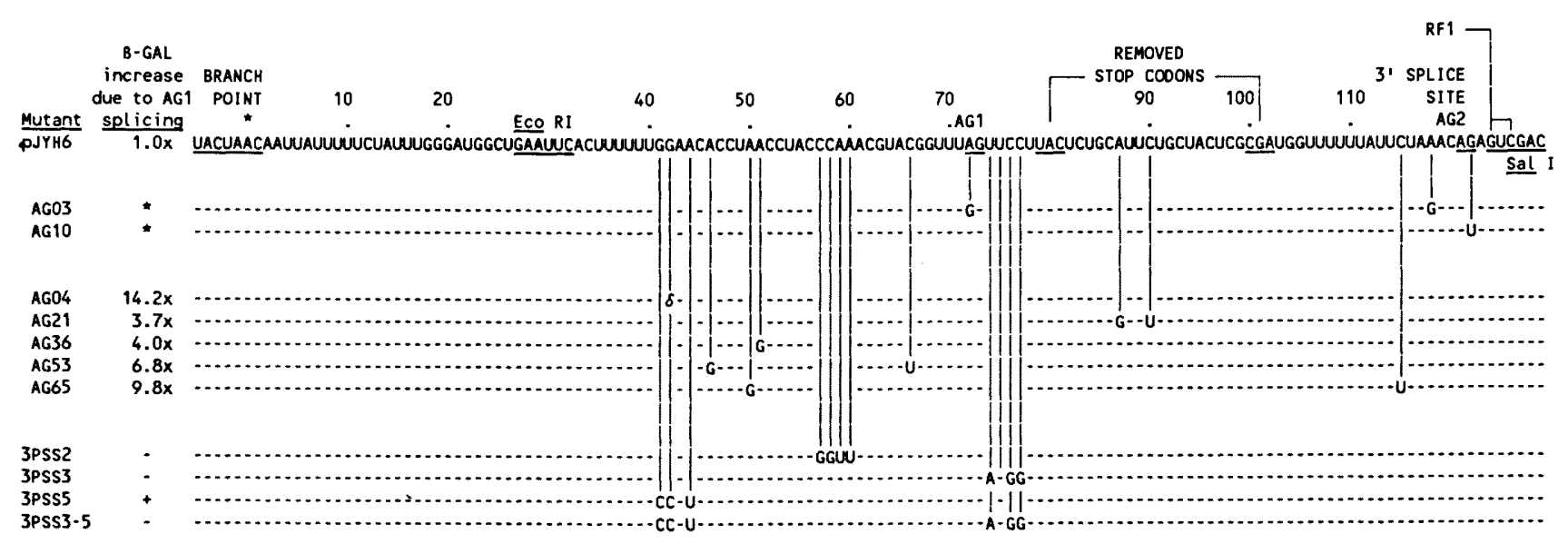

Figure 5. Mutations affecting 3 ' splice-site usage in the $S$. cerevisiae-K. lactis actin hybrid intron. The sequence of p/YH6 from the branchpoint to the Sall restriction site is shown at the top. Randomly induced mutations are signified by AG followed by a number. The relative extent of AGl activation as measured by $\beta$-gal activity is shown relative to pJYH6, which produces $1-3$ units of $\beta$-gal activity. Site-specific mutations are signified by 3PSS followed by a number. The asterisk $\left({ }^{*}\right)$ highlights the class I mutations (see text)

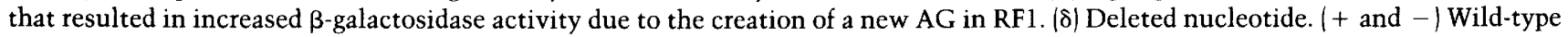
AG2 and AG1 levels of splicing, respectively, as determined by the X-gal plate assay.

Nuclease $\mathrm{S} 1$ analysis of the RNAs was used to verify $3^{\prime}$ splice-site preference seen for mutant AG04 using the PCR assay (Fig. 7). Nuclease S1 analysis of RNA prepared from a pJYH6 transformant shows that the GG is used to a much lesser extent than AG2 (Fig. 7, lane C), consistent with the PCR assay (Fig. 6). Mutant AG04, on the other hand, shows slight activation of AGl and virtually complete activation of the GG over AG2 (lane D). The combined results of the PCR assay and S1 analysis demonstrate that the point mutations, which occur in nonconserved sequences within the intron, can disrupt the overall pattern of $3^{\prime}$ splice-site selection.

The search for base-pair interactions reveals two possible structures that can explain how the proper $3^{\prime}$ splice site is selected

The computer program PCFOLD version 4.0 (Zucker and Steigler 1981) was used to identify potential secondary structures in the intron between the branchpoint and AG2, which could account for the skipping of AG1. An extensive secondary structure consisting of two domains separated by an interior loop (Fig. 8), located almost equidistant from the branchpoint and AG2, was predicted. The first domain consists of base-pair interactions 1833/84-98, whereas the second domain has base-pairing between 41-56/63-77, with the indicated bulges and loops. An alternative to the secondary structure (Fig. 8, right) is a potential pseudoknot (Fig. 8, left) in domain 2 of the hairpin just described. In this model the first domain of the secondary structure can still form (18-33/ 84-98|, but the second region folds differently. Two smaller stems are formed by the nucleotide interactions 48-56/63-73 and 57-59/40-42, making up the potential pseudoknot (Fig. 8). The most pleasing feature of the secondary and pseudoknot models is that they both seques- ter AG1 in helices while simultaneously positioning AG2 linearly 43 nucleotides downstream of the branchpoint, which is the average spacing found in yeast introns.

Each of the randomly isolated mutations described above falls into regions comprising either the hairpin or pseudoknot structures. Mutants AG04 (G41 deletion) and AG65 (A50 to G) disrupt the 40-42/57-59 interaction of the pseudoknot model and the 41-56/63-77 stem of the secondary model. Mutant AG36 (A51 to G) disrupts the 48-56/63-73 stem of the pseudoknot model and the 41-56/63-77 stem of the secondary model. AG21 (A87 to G; C90 to U) disrupts stem 18-33/84-98 of both models. AG53 (A46 to G; C66 to U) changes bulged nucleotides in both models. Most interesting is the position of the deleted guanine in mutant AG04, which could disrupt severely the short stem $(57-59 / 40-42)$ of the pseudoknot model. This stem is GC-rich and should stack nicely on top of the longer stem (48-56/63-73) with GC base pairs making for a stable pseudoknot. This could account for the fact that mutant AG04 has a stronger effect on 3' splice-site selection than any of the other mutations. Although the randomly isolated mutants do not allow differentiation between the pseudoknot and hairpin structural models, they do highlight a region of the pre-mRNA that has the potential to form secondary and possibly tertiary structure. Therefore, we tested directly the two alternative models by creating site-specific mutations.

\section{An extensive RNA hairpin is required for correct 3' splice-site selection}

Two mutations were created to differentiate between the two possible models (Fig. 8). 3PSS2 changes CCAA (5760 ) to GGUU, eliminating the $40-42 / 57-59$ stem of the 


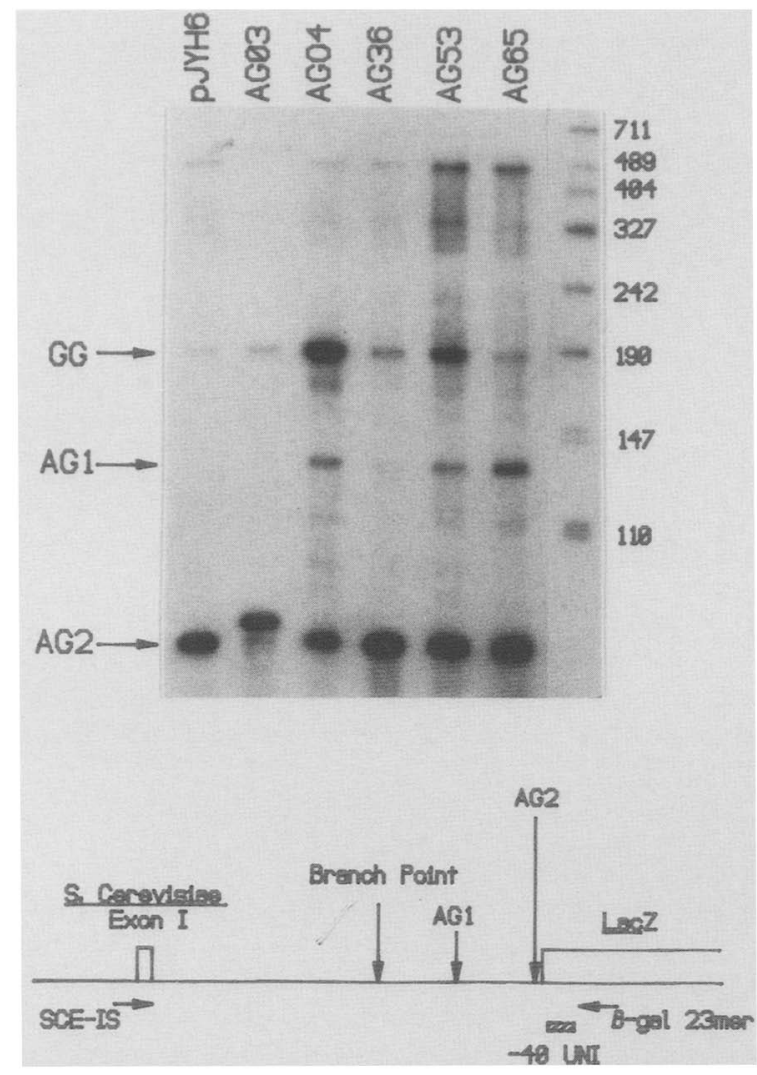

Figure 6. PCR analyses of $3^{\prime}$ splice-site selection in mutants. PCR analyses were carried out on 100 ng of total RNA from the indicated mutants as described in Materials and methods. Shown below is a diagram of the tripartite fusion gene (Fig. 4) and the primers used to determine the relative amounts of splicing to the GG, AG1, and AG2 splice sites. SCE-IS (5'-AATTAACAATGGATTCTG-3') includes the $S$. cerevisiae ACT exon I. The $\beta$-gal 23-mer $\left(5^{\prime}\right.$-TTAAGTTGGGTAACGCCAGGGTT- $3^{\prime}$ ) is complementary to lacZ. The probe -40 UNI (5'-GTTTTCCCAGTCACGAC-3') (Stratagene) is complementary to lac $Z$ and was used as a universal probe to detect all possible splices in the ACT-lac $Z$ fusion message. The products resulting from amplification of RNAs using the GG, AG1, and AG2 3' splice sites are indicated. The molecular weight marker is HpaII-digested pTZ18U (Bio-Rad).

pseudoknot model. This mutation does not disrupt the secondary structure and actually increases its stem by 1 bp. 3PSS3 changes UUCC (74-77) to AUGG, which only disrupts the secondary structure, leaving the potential pseudoknot intact. These mutations allow for discrimination between the two models because 3PSS2 disrupts only the pseudoknot model, whereas 3PSS3 disrupts only the hairpin model (Fig. 8). When JM43 was transformed with these mutant plasmids and scored for the LAC phenotype on X-gal plates, no activation of AG1 was detected (Fig. 5). The analysis of total RNA by the PCR assay, (Fig. 9), however, shows that 3PSS3 virtually eliminates usage of AG2 and activates the GG, whereas 3PSS2 maintains the parental pattern by preferring AG2 and using the GG to a lesser extent. These results sug- gest that the secondary structure is required for correct $3^{\prime}$ splice-site selection, because the overall pattern of $3^{\prime}$ splice-site selection is completely disrupted by the 3PSS3, and not the 3PSS2, mutations. In addition to the GG activation seen with the 3PSS3 mutation, a product that migrates slightly faster than the GG product and corresponds to a splice to a "new" GG located 5 nucleotides downstream of the GG at position $19 \mathrm{can}$ be observed. This product can be seen to a lesser extent with mutant AG04 (Fig. 5). Although this product could be an artifact of the PCR assay used, we believe it to be the result of a real splicing event to this new GG (see Discussion).

To prove that secondary interactions occur, one more site-specific mutant was created and is designated 3PSS5. 3PSS5 restores base-pairing in the 3PSS3 mutation in the secondary model by changing GGAA $(41-44)$ to CCAU (Fig. 10B). 3PSS5 was analyzed alone and in combination with 3PSS3. Surprisingly, this mutant alone directs most of the splicing to AG1 (Fig. 10A). However, when this mutant is combined with 3PSS3, a dramatic shift back to AG2 is seen (Fig. 10A). Interestingly, 3PSS5 has the potential to create a new, stable, stem-loop structure between the branchpoint and AGl (Fig. 10C). This potential stem-loop could position AG1 closer to the branchpoint, thereby allowing it to outcompete the GG as a $3^{\prime}$ splice site. The overall splicing efficiency in 3PSS 5 transformants, as judged by $\beta$-galactosidase activities on X-gal plates, is comparable to that of the pIYH6 transformants (Fig. 5).

\section{Discussion}

We have isolated several point mutations that activate a quiescent PyAG as a $3^{\prime}$ ' splice signal in the $K$. lactis ACT

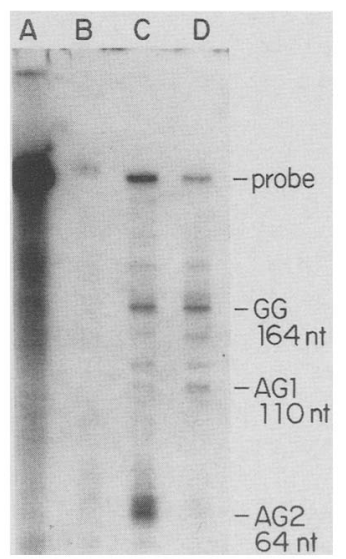

Figure 7. Nuclease $S 1$ analyses confirm the preference of $3^{\prime}$ splice sites in p/YH6 and mutant AG04. Nuclease S1 analyses of total RNAs were performed as described in Materials and methods. (Lane $A$ ) No S1 nuclease control; (lane $B$ ) RNA from untransformed $S$. cerevisiae; (lane $C$ ) RNA from $S$. cerevisiae transformed with pJYH6; (lane D) RNA from mutant AG04. Protected products resulting from splicing to the GG, AG1, and AG2 are indicated with their molecular sizes. 

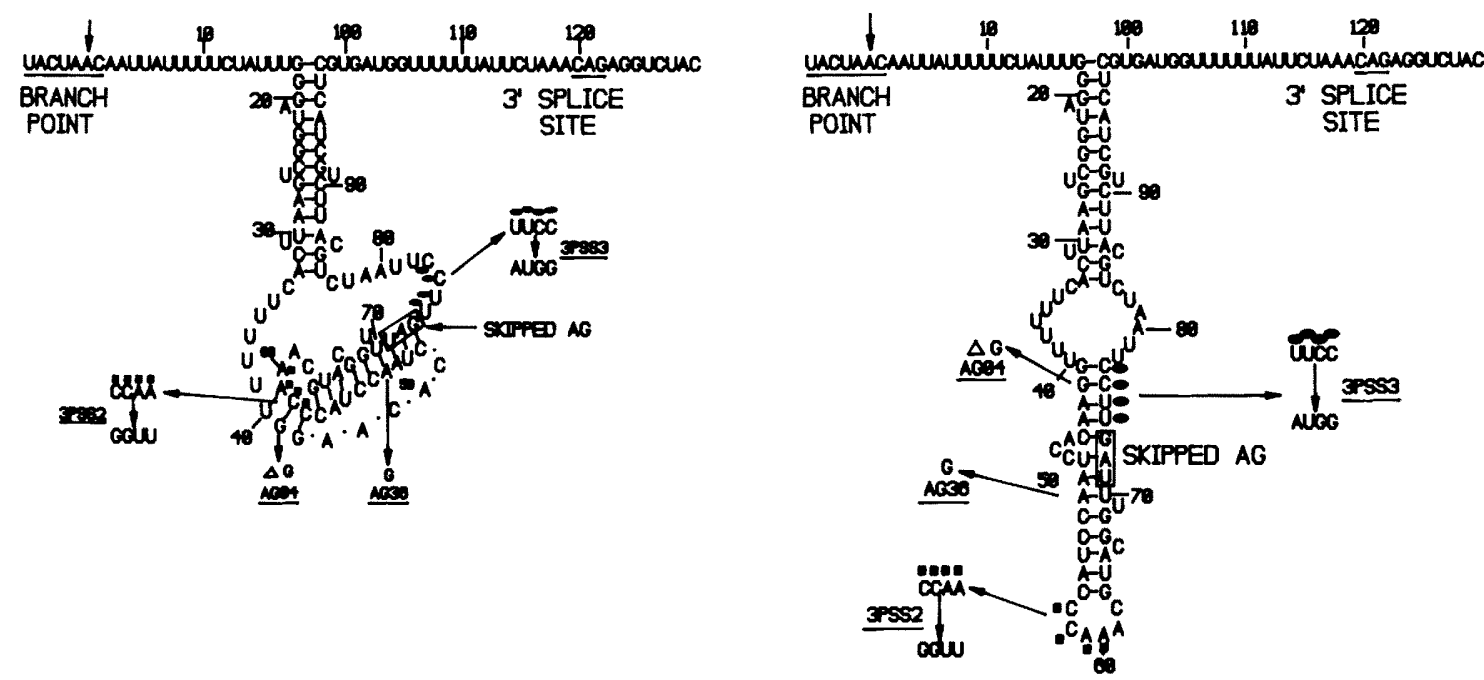

Figure 8. Two possible structures can form between the branchpoint and AG2. A computer-predicted potential pseudoknot structure (left) and a secondary a structure (right) are shown. The numbering in both models is consistent with previous figures, and selected mutations are shown in both models.

gene. In addition to activating this PyAG, some of the mutants also activate another competing 3 ' splice site (GG at position 19). We favor a structural model as opposed to a model involving sequence-specific binding of a spliceosomal factor(s) to account for proper 3' splicesite selection in the $K$. lactis ACT intron for the following reasons: (1) Mutations on both sides of AGl cause activation; (2) the mutations cluster in a region that has folding potential; $(3)$ the sequences in this region fit no known consensus sequences; (4) mutant AG04 (a single nucleotide deletion) severely affects usage of the GG, AG1, and AG2. It would be more likely that a single-

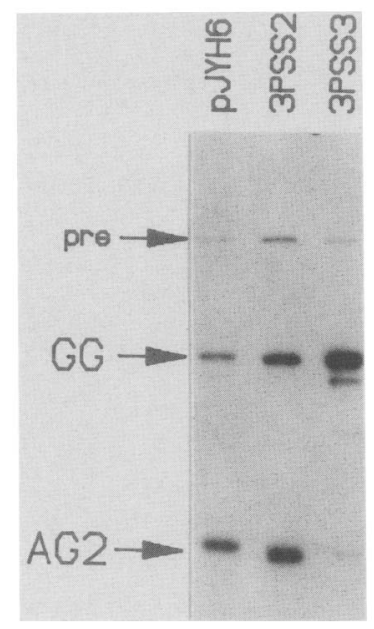

Figure 9. A mutation disrupting the secondary structure, not the pseudoknot, disrupts the normal pattern of $3^{\prime}$ splice-site selection. PCR analyses were done as described in Fig. 6 on 100 ng of total RNA prepared from the indicated mutants, which are also shown in Fig. 8. Amplified products corresponding to precursor and splicing to the GG and AG2 are indicated. nucleotide change affects the overall structure of the intron as opposed to altering the relative binding of a spliceosomal factor(s) to three competing $3^{\prime}$ splice sites because cryptic splice sites are not usually activated with point mutations in yeast.

Genetic testing of two different folding models suggests that secondary interactions are required for correct processing of this actin intron; the strongest disrupter of the putative secondary structure, mutant 3PSS3 (Fig. 8), completely interferes with the normal pattern of $3^{\prime}$ splice-site selection (see Figs. 9 and 10A). Analyses of RNA from mutant 3PSS3 also suggests that a severe disruption of the secondary structure forces most of the splicing to the GG without activating AGl. This is evidenced by the fact that 3PSS3 utilizes the GG almost exclusively (Figs. 9 and 10A) and does not activate AG1 as indicated by the X-gal plate assay (Fig. 5). Finally, analysis of the compensatory mutation 3PSS3/3PSS5, which restores base-pairing in the stem of Figure 10B, proves that secondary base-pairing interactions are required for AG2 utilization.

\section{How do the point mutations activate AG1?}

The data presented suggest that it may not be possible to fully activate AG1 in the presence of the two competing sites with a single point mutation. The genetic screen employed facilitates the isolation of a special class of mutations that activate the least favored of three splice sites. Full activation of AGl appears to occur only with mutant 3PSS5, which has the potential to position AG1 closer to the branchpoint by forming a new hairpin structure between the branchpoint and AG1 (Fig. 10C). How, then, do the point mutations elicit their effects on both AGl and the GG at position 19? The sequences of the randomly induced AG1-activating mutants were folded 

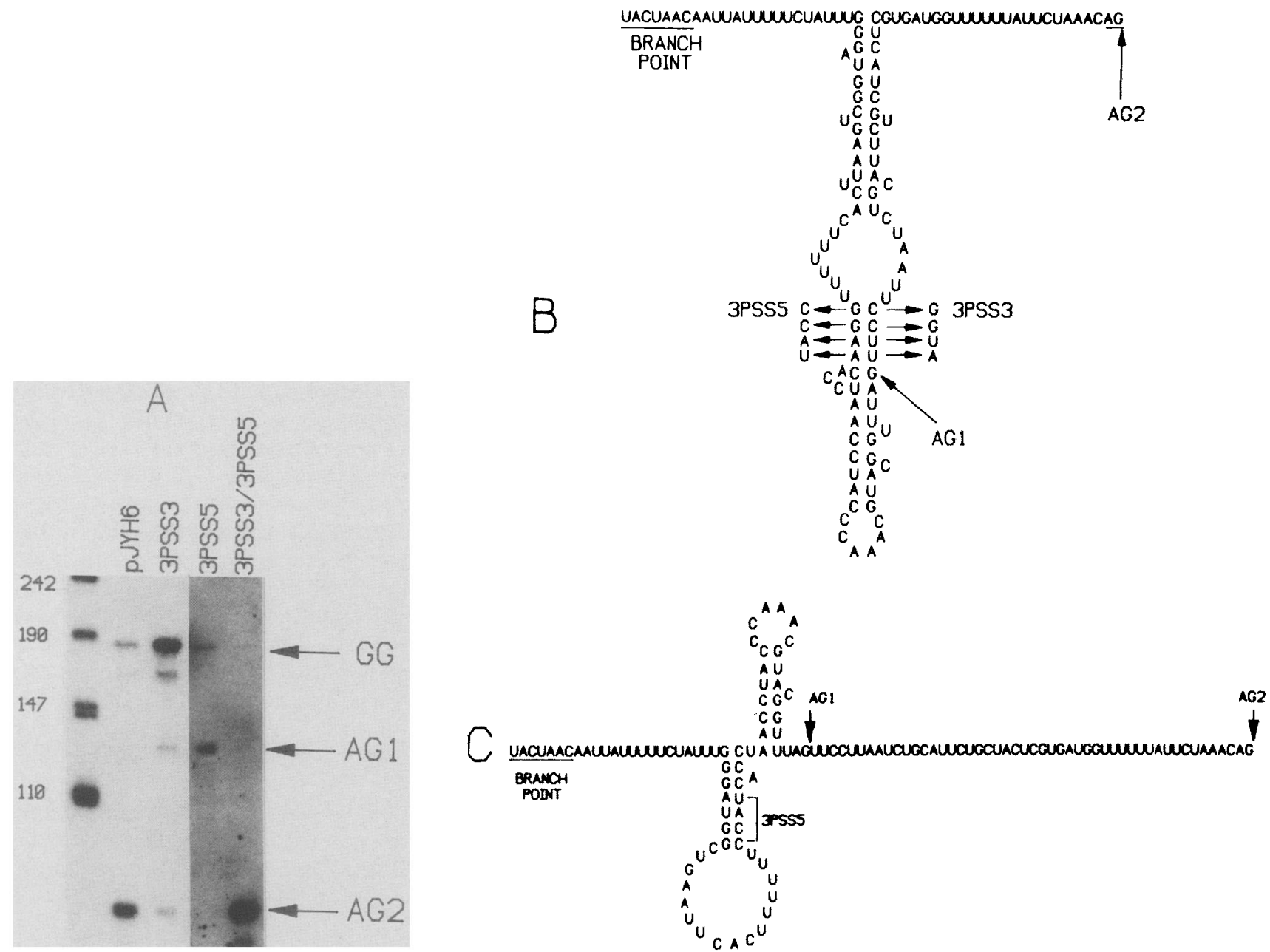

Figure 10. Compensatory mutations in the ACT intron secondary structure restore splicing. $(A)$ PCR analyses were done as described in Fig. 6 on the indicated mutants. $(B)$ The sequence of the mutations and their relative positions in the secondary structure. $(C)$ Representation of a new computer-predicted hairpin created by the 3PSS5 mutation, showing the position of AGI relative to the branchpoint.

using the computer program PCFOLD (Zucker and Steigler 1981). With the exception of mutant AG21, all form the original hairpin structure with only minor alterations. The predicted free energies of the mutant structures, however, are weaker than the wild type $1-13.9$ kcal), except for mutant AG53. Interestingly, a decrease in stability of the hairpin corresponds to an increased activation of AG1 on the basis of $\beta$-galactosidase activity. The free energies of mutants AG04 (14× activation),

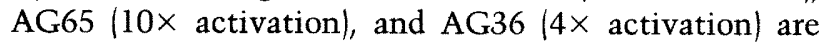
$-10.8,-13.1$, and $-13.3 \mathrm{kcal}$, respectively. These mutations are all relatively close to AG1 (within $3 \mathrm{bp}$ ) in the hairpin structure. Therefore, it is possible that they increase local breathing of the hairpin close to AG1, leaving it more exposed to the splicing machinery. The fact that the double mutant AG53 activates AG1 without altering any base pairs of the secondary structure implies that bulging nucleotides may also contribute to the overall stability and/or the three-dimensional structure of the precursor RNA. In short, it appears that local breathing of the hairpin and/or the three-dimensional orientation of AG1 within this structure are the factors most likely causing its activation as a $3^{\prime}$ splice site. Mutant AG21 does not form the wild-type secondary structure when analyzed with PCFOLD, and we have no explanation for the fourfold activation of AGl seen with this mutant.

Two of the mutants analyzed (AG04 and 3PSS3) show high activation of the GG at position 19. Mutant AG04, which is the strongest activator of AG1, utilizes the GG to a higher extent than any of the other randomly isolated mutations. Mutant AG04 also forms the weakest secondary structure of the point mutants. A more strongly disrupting mutation, 3PSS3, results in complete activation of this GG. Thus, it appears that in the absence of the secondary structure, the GG at position 19 is activated and becomes the preferred $3^{\prime}$ splice site / see below). 
What are the implications for a mechanism of $3^{\prime}$ splice-site selection?

A molecular scanning model has been proposed in mammalian systems to account for $3^{\prime}$ splice-site selection (Smith et al. 1989). In this model the spliceosome scans downstream of the branchpoint and splices the upstream exon adjacent to the first AG encountered. Smith and colleagues (1989) demonstrated that the scanning process is blocked by secondary structures inserted between the branchpoint and 3' splice site. Inspection of the $K$. lactis ACT intron sequence tells us that such a simple scanning model cannot account for the observed splicing pattern. A situation similar to that observed here is seen with the TUB3 gene of $S$. cerevisiae (Schatz et al. 1986), which contains unusually long spacing between the branchpoint and the $3^{\prime}$ splice site and in which several unused AG dinucleotides precede the 3 ' splice site. Perhaps higher-order structure and/or the position of polyuridine tracts (Patterson and Guthrie 1991) determine 3' splice-site selection in this gene. It appears, however, that regional scanning does occur in some sequence contexts. For instance, mutant AG03 has a new AG introduced just 5 nucleotides upstream of AG2. Most of the splicing uses this new $A G$, whereas very little splicing goes to AG2 (Fig. 6). A similar situation is noted for the 3PSS3 mutation and somewhat for mutant AG04. Both prefer the GG (position 19) as a $3^{\prime}$ splice site but also produce slightly detectable amounts of a unique product that corresponds to a splice to another GG located 5 nucleotides downstream of the GG (position 19). It is possible that removal of the GG (position 19) would activate this downstream GG to high levels, which would support the notion that this region of the intron is poised for $3^{\prime}$ splice-site selection in $S$. cerevisiae.

No point mutations were isolated that activate AGl to wild-type levels. We attribute this to the poor proximity of AG1 with respect to the branchpoint (73 nucleotides), the GG at position 19, and AG2. Thus, it is apparent that 3 ' splice sites are influenced by the overall sequence context and/or relative position with respect to the branchpoint. In this regard, an AG $\rightarrow$ GG (Fouser and Friesen 1987) mutation in the $S$. cerevisiae ACT $3^{\prime}$ splice site still allows accurate splicing to occur. Also, the presence of an upstream polyuridine tract increases the usage of competing alternative $3^{\prime}$ splice sites (Patterson and Guthrie 1991). It should be noted that a large polyuridine tract is present upstream of the GG dinucleotide at position 19 (see Fig. 1), perhaps accounting, in part, for its efficient utilization in $S$. cerevisiae. Finally, it seems that positioning AG1 closer to the branchpoint with the new hypothetical hairpin of mutant 3PSS5 (Fig. 10C) enables it to out-compete the GG as a 3 ' splice site, further demonstrating that there is competition between $3^{\prime}$ splice sites; this competition can be controlled by RNA folding. These results demonstrate a positioning requirement that is critical in determining the choice of splice acceptor sites. At this point, it becomes tempting to speculate that some forms of alternative splicing in metazoans may have evolved from a simple mechanism of RNA folding to the more complicated use of proteins that interact with RNA secondary structures to influence the choice of alternative splice sites.

\section{How did this structural motif evolve?}

Visual inspection of the secondary structure in Figure 8 implies that removal of the complete hairpin structure from this ACT intron would make AG2 conform to the characteristics of a typical yeast $3^{\prime}$ splice site. An average spacing of 43 nucleotides would separate the branchpoint and 3 ' splice site, with a polyuridine tract just upstream of AG2. Therefore, we hypothesized that an insertion sequence (IS) element might have integrated between the branchpoint and 3 ' splice site of the K. lactis ACT gene in such a way that splicing could proceed in a relatively uninterrupted fashion. We searched for sequence similarities to IS elements and transposons in GenBank using the FASTDB program (Intelligenetics). A curious and provocative homology was found to the right terminal inverted repeat (RTIR) of Tn1000. The Tn1000 RTIR contains 16 of 18 nucleotides identical to the putative IS element in the ACT intron (Fig. 11). Such identity would be expected to occur only by random chance once in every $1.4 \times 10^{7}$ nucleotides. This finding becomes even more intriguing if we consider that $E$. coli $\mathrm{F}$ factor can be transferred to yeast (Heinemann and Sprague 1989) and that $\operatorname{Tn} 1000$ is an F-factor component in E. coli (Saadi et al. 1987). Although we cannot state that a prokaryotic IS was the source of this particular intron element, it is likely that introns can and do absorb

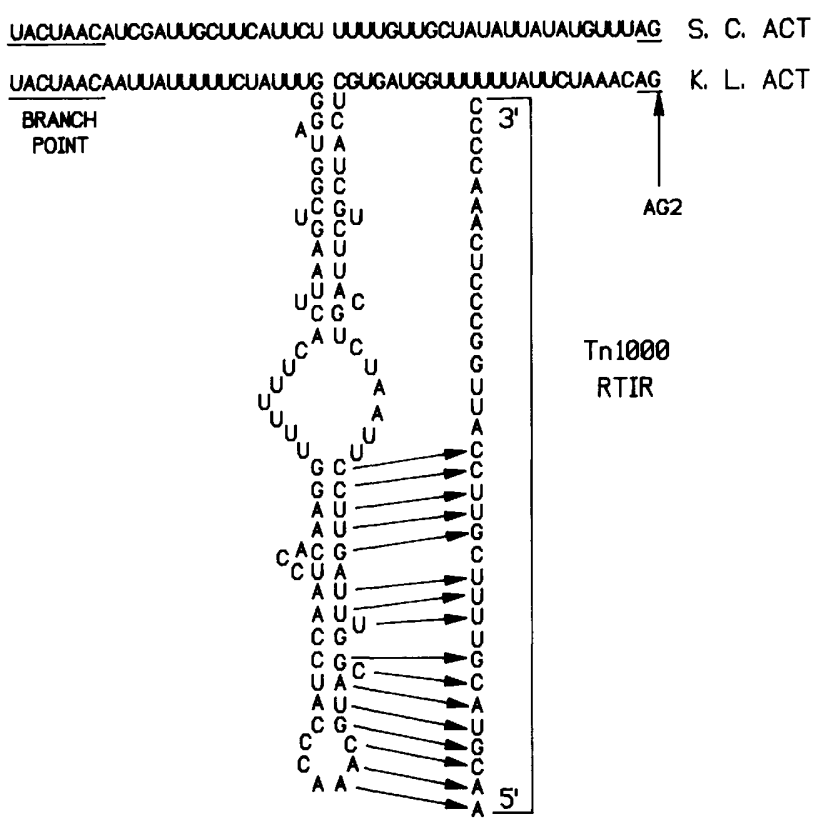

Figure 11. K. lactis ACT 3' splice site shows homology to the bacterial Tn1000 RTIR. The RNA sequences of the $S$. cerevisiae ACT 3' splice site, K. lactis 3' splice site, and complete Tn 1000 RTIR are shown with the homologous nucleotides indicated by arrows. 
sequences that do not inhibit the splicing process. These IS elements might persist by folding into nondisruptive structures such as the one described for the sequence in the $K$. lactis ACT intron.

\section{Materials and methods}

Strains, plasmid constructions, and transformations

K. lactis strain 5D298 (lac4-8, ura3-3, ade3-1) and S. cerevisiae strain JM43 (leu2, ura3, trp1, his3), kindly provided by M. Riley and $\mathrm{J}$. McEwen, respectively, were used throughout these studies. Both were transformed using the lithium acetate method (Ito et al. 1983). Plasmid pCVBB has been described previously (Deshler et al. 1989) and contains the 2.6-kb BamHI-BgIII fragment of the $K$. lactis ACT gene including $1.5 \mathrm{~kb}$ of the $5^{\prime}$-untranslated region, exon $I$, the complete intron, and $\sim 240$ nucleotides of exon II.

Plasmids pJYH6 and pJYH7 were constructed in a series of site-specific mutagenesis and subcloning steps. First the $2.6-\mathrm{kb}$ BamHI-BglII fragment of the K. lactis ACT gene was cloned into the Bluescript $\mathrm{KS} \mathrm{M}^{+} 3^{+}$plasmid vector (Stratagene). Oligonucleotides oAG3 (5'-TCTAAACAGAGTCGACTGCT-3') and oAG1 $\left(5^{\prime}\right.$-CAGAGGTCGACTGCTTTAG-3' $)$ were then used to create a Sall site 1 or 2 nucleotides, respectively, downstream of the 3 ' splice site using in vitro mutagenesis (see Fig. 1). The stop codons were altered with a single mutagenic nucleotide, oAG4 (5'-TAGTTCCTTACTCTGCATTCTGCTACTCGCGATGGTTTTT-3'). The resulting 2.4-kb BamHI-SaII fragments were cloned upstream of the $l a c Z$ gene in an $S$. cerevisiae $2 \mu$, URA3 shuttle vector p2UB (G.P. Larson and J.J. Rossi, unpubl.), creating two K. lactis ACT-E. coli lacZ fusion genes. Next, a PCR reaction was used to amplify the $S$. cerevisiae ACT gene $\left(\mathrm{Ng}\right.$ and Abelson 1980) using a $5^{\prime}$ primer that includes the BamHI site upstream of the promoter $15^{\prime}$-TATCGGATCCTCAAAACCCT-3') and a "fusion" 3 ' primer that includes the UACUAAC sequence (5'-AAGTGAATTCAGCCATCCCAAATAGAAAAATAATTGTTAGTACATGAGACT- $3^{\prime}$ ). The $5^{\prime} 35$ nucleotides of this primer are complementary to the $K$. lactis ACT intron downstream of the branchpoint and include the EcoRI site (Fig. 1), whereas the 3' 16 nucleotides are complementary to the $S$. cerevisiae UACUAAC and adjoining upstream 9 nucleotides. After 30 cycles of amplification, the PCR product was digested with $B a m H I$ and $E c o$ RI and cloned in place of the analogous BamHI-EcoRI, K. lactis ACT DNA fragment in the two $K$. lactis ACT-E. coli lacZ fusion genes just described.

\section{DNA manipulations and random mutagenesis}

Restriction endonucleases, T4 DNA ligase, and T4 kinase were purchased from Bethesda Research Laboratories. Nucleotides labeled with ${ }^{32} \mathrm{P}$ were purchased from DuPont. Avian myeloblastosis virus (AMV) reverse transcriptase was obtained from Life Sciences (St. Petersburg, FL).

Site-specific mutagenesis was done either with the Bio-Rad or U.S. Biochemical mutagenesis kit. The mutagenic oligonucleotides were purchased from GENOSYS (The Woodlands, TX) or were synthesized by the City of Hope DNA synthesis facility.

Random mutagenesis of the K. lactis ACT 3' splice site was performed as follows. The 97-nucleotide EcoRI-SalI fragment of pJYH6 was cloned in opposite orientations into the vectors M13mp8 and M13mp9. Single-stranded DNAs were purified, and $40 \mu \mathrm{g}$ of each clone was incubated with hydrazine, formic acid, and nitrous acid as described (Ausubel et al. 1990). After ethanol precipitation, all DNAs were pooled and $0.1 \mu \mathrm{g}$ of the mixture was amplified in a PCR using the universal M13 sequencing primer and a reverse sequencing primer that flanked the polylinker. The first two rounds of denaturation, annealing, and polymerization were carried out with 1 unit of freshly added AMV reverse transcriptase, and the subsequent 32 cycles, with Taq DNA polymerase (Perkin Elmer Cetus). Aliquots $(2-3 \mu \mathrm{g}$ ) were obtained from the reaction and were subsequently digested with EcoRI and Sall. The resulting 97-nucleotide fragments /containing the chemically induced sequence heterogeneity) were gel-purified from a $6 \%$ polyacrylamide gel and cloned between the TACTAAC and lacZ gene of pJYH6. These clones were transformed into $E$. coli strain MC1061 (Casabadan and Cohen 1980). Sequencing of randomly picked clones indicated that $\sim 10 \%$ of the target molecules contained a mutation. Plasmid DNA was extracted from 10,000 pooled $E$. coli transformants and was used to transform $S$. cerevisiae strain JM43. Transformants were initially plated onto yeast synthetic minimal media plates (Sherman et al. 1986) and subsequently patched onto similar plates containing $0.004 \% \mathrm{X}$-gal and $1 \times \mathrm{M} 9$ salts (Miller 1972). Transformants harboring pJYH6 and pJYH7 were always included on the plates as the controls for low and high $\beta$-galactosidase-expressing transformants.

\section{RNA analysis}

Yeast transformants were grown in $10 \mathrm{ml}$ of yeast synthetic minimal media (Sherman et al. 1986) to an $\mathrm{OD}_{600}$ of 1.0. Total RNA was prepared from these cultures as described by Domdey et al. (1984).

Amplification of specific mRNA species was performed as described previously (Saiki et al. 1988; Deshler et al. 1989) using primers described in the figure legends. The DNA products derived from the RNA-dependent PCR were electrophoresed in an $8 \mathrm{~m}$ urea- $-6 \%$ polyacrylamide gel. Following electrophoresis, the products were transferred to a Zetaprobe (Bio-Rad) nylon membrane using a Bio-Rad electrotransfer device in $1 \times$ TAE at $1 \mathrm{amp}$ for $\geqslant 3 \mathrm{hr}$. The Zetaprobe filter was prehybridized in $6 \times$ SSPE, $7 \%$ SDS, and $0.5 \%$ nonfat dried milk (Carnation) at $55^{\circ} \mathrm{C}$ for $1 \mathrm{hr}$ and then hybridized with a ${ }^{32} \mathrm{p}$-labeled oligonucleotide probe in the same buffer at the same temperature for $\geqslant 3 \mathrm{hr}$. The filters were washed twice at $55^{\circ} \mathrm{C}$ in $6 \times$ SSC and autoradiographed. The compositions of all buffers used in these studies are described in Maniatis et al. (1982).

The probe used for $\mathrm{S} 1$ nuclease analysis was generated by kinasing, with $\left[\gamma^{-}{ }^{32} \mathrm{P}\right]$ ATP, 10 pmoles of the $\beta$-gal 23 -mer (see Fig. 6), and using it in a PCR in conjunction with 1 pmole of unlabeled SCE-IS oligonucleotide (see also Fig. 6) to amplify DNA from the pIYH6 plasmid template. The product was purified following electrophoresis on an $8 \mathrm{M}$ urea- $6 \%$ polyacrylamide gel, and one-tenth of the recovered product was annealed to $\sim 5 \mu \mathrm{g}$ of total RNA in $30 \mu \mathrm{l}$ of hybridization buffer (Berk and Sharp 1977) at $45^{\circ} \mathrm{C}$ for $3 \mathrm{hr}$. S1 buffer $(300 \mu l)$ (Maniatis et al. 1982) containing $S 1$ nuclease at a concentration of $200 \mathrm{U} / \mu \mathrm{l}$ was added, and the reactions were incubated at $30^{\circ} \mathrm{C}$ for $60 \mathrm{~min}$. The reactions were stopped with $50 \mu \mathrm{l}, 4 \mathrm{M} \mathrm{NH}_{4} \mathrm{OAc}$, and $0.1 \mathrm{M}$ EDTA, extracted with phenol, EtOH-precipitated, and electrophoresed in an $8 \mathrm{M}$ urea- $6 \%$ polyacrylamide gel. The gel was dried and subsequently autoradiographed.

\section{Nucleic acid sequencing}

Sequencing of the 118-nucleotide PCR GG product (Fig. 2A, lane 4) was done as follows: The product was visualized with ethidium bromide, eluted, and asymmetrically reamplified using the primers described in the legend to Figure 2, except that 
SCE-IS was in 25-fold molar excess over the other primer to generate single-stranded DNA for sequence analysis. The product from this reamplification was gel-purified and sequenced with the U.S. Biochemical Sequenase kit using the KL3'IVS /see legend to Fig. 2) oligonucleotide as a primer and $\alpha^{-35} \mathrm{~S}$-labeled dATP.

Sequencing of mutants exhibiting increased $\beta$-galactosidase activities (see Fig. 5) was done by first purifying total DNA from $5-\mathrm{ml}$ cultures of the yeast transformants (Sherman et al. 1986), transforming $E$. coli with the yeast DNA, and selecting for ampicillin resistance. Plasmid DNA was then purified from the E. coli transformants and sequenced as described previously (Hattori and Sakaki 1986) with the Sequenase kit (U.S. Biochemical) using the ${ }^{32}$ P-labeled $\beta$-gal 23-base oligonucleotide (see Fig. 6) complementary to the amino-terminal region of the lac $Z$ gene described in the text.

\section{$\beta$-Galactosidase assays}

$\beta$-Galactosidase assays were done a minimum of three times, in duplicate. This method has been described previously (Cellini et al. 1986).

\section{Acknowledgments}

We thank Daniela Castanotto, Pete Gertson, Dennis Arvidson, and Gayle Knapp for helpful and stimulating discussions during this project. This work is in partial fullfilment of the Ph.D. degree for J.D., and was supported by grants from the IDEN Foundation, National Institute of Allergy and Infectious Diseases (5R01AI293292D02), and a grant given in honor of Tom Bane from the National Lupus Erythematosus Foundation. J.R. is a member of the Cancer Center (support grant CA 33572).

The publication costs of this article were defrayed in part by payment of page charges. This article must therefore be hereby marked "advertisement" in accordance with 18 USC section 1734 solely to indicate this fact.

\section{References}

Ausubel, F.A., R. Brent, R.E. Kingston, D.D. Moore, J.G. Seidman, J.A. Smith, and K. Struhl. 1990. Current protocols in molecular biology. Greene Publishing/Wiley-Interscience, New York.

Berk, A.J. and P.A. Sharp. 1977. Sizing and mapping of early adenovirus mRNAs by gel electrophoresis of S1 endonuclease-digested hybrids. Cell 12: 721-732.

Casabadan, M.J. and S. Cohen. 1980. In vitro gene fusions that join an enzymatically active $\beta$-galactosidase segment to amino-terminal fragments of exogenous proteins: Escherichia coli plasmid vectors for the detection and cloning of translational signals. I. Bacteriol. 143: 971-980.

Cellini, A., E. Felder, and J.J. Rossi. 1986. Yeast pre-messenger RNA splicing efficiency depends on critical spacing requirements between the branch point and $3^{\prime}$ splice site. EMBO $J$. 5: 1023-1030.

Chebli, K., R. Gattoni, P. Schmitt, G. Hildwein, and J. Stevenin. 1989. The 216-nucleotide intron of the E1A pre-mRNA contains a hairpin structure that permits utilization of unusually distant branch acceptors. Mol. Cell. Biol. 9: 4852-4861.

Deshler, J.O., G.P. Larson, and J.J. Rossi. 1989. Kluyveromyces lactis maintains Saccharomyces cerevisiae intron-encoded splicing signals. Mol. Cell. Biol. 9: 2208-2213.

Domdey, H., B. Apostol, R.J. Lin, A. Newman, E. Brody, and J. Abelson. 1984. Lariat structures are in vivo intermediates in yeast pre-mRNA splicing. Cell 39: 611-621.

Eperon, L.P., I.R. Graham, A.D. Griffiths, and I.C. Eperon. 1988. Effects of RNA secondary structure on alternative splicing of pre-mRNA: Is folding limited to a region behind the transcribing RNA polymerase? Cell 54: 393-401.

Fouser, L.A. and J.D. Friesen. 1987. Effects on mRNA splicing of mutations in the $3^{\prime}$ region of the Saccharomyces cerevisiae actin intron. Mol. Cell. Biol. 7: 225-230.

Hattori, M. and Y. Sakaki. 1986. Dideoxy sequencing method using denatured plasmid templates. Anal. Biochem. 152: 232-238.

Heinemann, J.A. and G.F. Sprague, Jr. 1989. Bacterial conjugative plasmids mobilize DNA transfer between bacteria and yeast. Nature 340: 205-209.

Helfman, D.M., R.F. Roscigno, G.J. Mulligan, L.A. Finn, and K.S. Weber. 1990. Identification of two distinct intron elements involved in alternative splicing of $\beta$-tropomyosin premRNA. Genes \& Dev. 4: 98-110.

Ito, H., Y. Fukuda, K. Murata, and A. Kimura. 1983. Transformation of intact yeast cells treated with alkali cations. $J$. Bacteriol. 153: 163-168.

Langford, C.J. and D. Gallwitz. 1983. Evidence for an introncontained sequence required for the splicing of yeast RNA polymerase II transcripts. Cell 33: 519-2D527.

Libri, C., M. Goux-Pelletan, E. Brody, and M.Y. Fiszman. 1990. Exon as well as intron sequences are cis-regulating elements for the mutually exclusive alternative splicing of the $\beta$ tropomyosin gene. Mol. Cell. Biol. 10: 5036-2D5046.

Maniatis, T., E.F. Fritsch, and J. Sambrook. 1982. Molecular cloning: A laboratory manual. Cold Spring Harbor Laboratory, Cold Spring Harbor, New York.

Miller, J.H. 1972. Experiments in molecular genetics. Cold Spring Harbor Laboratory, Cold Spring Harbor, New York.

Nasim, F.H., P.A. Spears, H.M. Hoffman, H. Kuo, and P.J. Grabowski. 1990. A sequential splicing mechanism promotes selection of an optional exon by repositioning a downstream $5^{\prime}$ splice site in preprotachykinin pre-mRNA. Genes \& Dev. 4: 1172-1184.

Newman, A. 1987. Specific accessory sequences in Saccharomyces cerevisiae introns control assembly of pre-mRNAs into spliceosomes. $E M B O$ J. 6: 3833-3839.

$\mathrm{Ng}$, R. and J. Abelson. 1980. Isolation and sequence of the gene for actin in Saccharomyces cerevisiae. Proc. Natl. Acad. Sci. 77: 3912-3916.

Padgett, R.A., M. Konarska, P.J. Grabowski, S.F. Hardy, and P.A. Sharp. 1984. Lariat RNAs as intermediates and products in the splicing of messenger RNA precursors. Science 225: 898-903.

Parker, R., P.G. Siliciano, and C. Guthrie. 1987. Recognition of the TACTAAC box during mRNA splicing in yeast involves base pairing to the U2-like snRNA. Cell 49: 229-239.

Patterson, B. and C. Guthrie. 1991. A U-rich tract enhances usage of an alternative $3^{\prime}$ splice site in yeast. Cell 64: 181187.

Pikielny, C.W. and M. Rosbash. 1985. mRNA splicing efficiency in yeast and the contribution of non-conserved sequences. Cell 41: 119-126.

Reed, R. and T. Maniatis. 1986. A role for exon sequences and splice-site proximity in splice-site selection. Cell 46: 681690.

Ruskin, B., A.R. Krainer, T. Maniatis, and M.R. Green. 1984. Excision of an intact intron as a novel lariat structure during pre-mRNA splicing in vitro. Cell 38: 317-331.

Rymond, B.C. and M. Rosbash. 1985. Cleavage of $5^{\prime}$ splice site and lariat formation are independent of $3^{\prime}$ splice site in yeast mRNA splicing. Nature 317: 735-737. 
Saadi, S., W.K. Maas, D.F. Hill, and P.L. Bergquist. 1987. Nucleotide sequence analysis of RepFIC, a basic replicon present in IncFI plasmids $\mathrm{P} 307$ and $\mathrm{F}$, and its relation to the repA replicon of IncFII plasmids. $/$. Bacteriol. 169: 18361846.

Saiki, R.K., D.H. Gelfand, S. Stoffel, S.J. Scharf, R. Higuchi, G.T. Horn, K.B. Mullis, and H.A. Erlich. 1988. Primer-directed enzymatic amplification of DNA with a thermostable DNA polymerase. Science 239: 487-491.

Schatz, P.J., L. Pillus, P. Grisifi, F. Solomon, and D. Botstein. 1986. Two functional $\alpha$-tubulin genes of the yeast Saccharomyces cerevisiae encode divergent proteins. Mol. Cell. Biol. 6: 3711-3721.

Seraphin, B., L. Kretzner, and M. Rosbash. 1988. A U1 snRNA : Pre-mRNA base pairing interaction is required early in yeast spliceosome assembly but does not uniquely define the 5' cleavage site. EMBO I. 7: 2533-2538.

Sherman, F., G.R. Fink, and J.B. Hicks. 1986. Methods in yeast genetics. Cold Spring Harbor Laboratory, Cold Spring Harbor, New York.

Siliciano, P.G. and C. Guthrie. 1988. 5' Splice site selection in yeast: Genetic alterations in base-pairing with U1 reveal additional requirements. Genes \& Dev. 2: 1258-1267.

Smith, C.W.J., E.B. Porro, J.G. Patton, and B. Nadal-Ginard. 1989. Scanning from an independently specified branch point defines the $3^{\prime}$ splice site of mammalian introns. $\mathrm{Na}$ ture 342: 243-247.

Solnick, D. 1985. Alternative splicing caused by RNA secondary structure. Cell 43: 667-676.

Vijayraghavan, U., R. Parker, J. Tamm, Y. Iimura, J. Rossi, J. Abelson, and C. Guthrie. 1986. Mutations in conserved intron sequences affect multiple steps in the yeast splicing pathway, particularly assembly of the spliceosome. EMBO J. 5: 1683-1695.

Yoshimatsu, T. and F. Nagawa. 1989. Control of gene expression by artificial introns in Saccharomyces cerevisiae. Science 244: 1346-1348.

Zeitlin, S. and A. Efstratiadis. 1984. Introns splicing products of rabbit beta-globin pre-mRNA. Cell 39: 589-602.

Zucker, M. and P. Steigler. 1981. Optimal computer folding of large RNA sequences using thermodynamics and auxiliary information. Nucleic Acids Res. 9: 133-148. 


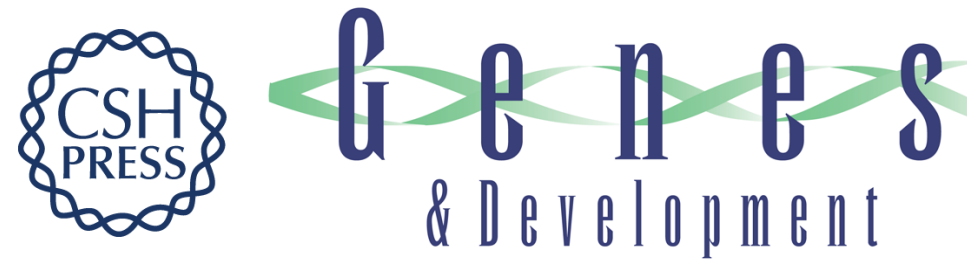

\section{Unexpected point mutations activate cryptic 3' splice sites by perturbing a natural secondary structure within a yeast intron.}

J O Deshler and J J Rossi

Genes Dev. 1991, 5:

Access the most recent version at doi:10.1101/gad.5.7.1252

References This article cites 35 articles, 15 of which can be accessed free at: http://genesdev.cshlp.org/content/5/7/1252.full.html\#ref-list-1

License

Email Alerting

Service

Receive free email alerts when new articles cite this article - sign up in the box at the top right corner of the article or click here.

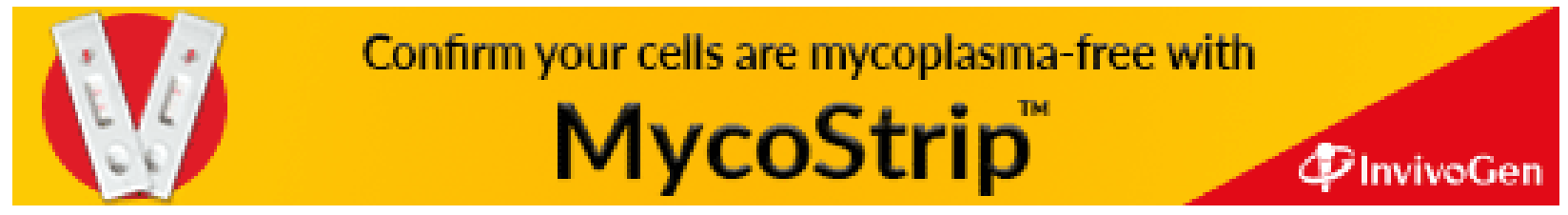

